\title{
Docosapentaenoic acid (omega-3): is it a reservoir of EPA in mammals?
}

\author{
G. Kaur and A. J. Sinclair \\ Metabolic Research Unit, School of Medicine, Deakin University, Waurn Ponds, Victoria 3217, Australia
}

Long chain omega 3 polyunsaturated fats play an important role in brain and cardiovascular function, and in reducing pain and inflammation in conditions such as rheumatoid arthritis. EPA is regarded as important for inflammation and cardiovascular function, while DHA is regarded as crucial for brain function. The role of docosapentaenoic acid (DPA) is unknown. Many studies have shown cardiovascular, arthritis relief or neural benefits of consuming fish or fish oils which typically contain all three of these omega 3 fatty acids. In addition, EPA and DHA have been studied as individual compounds and shown to contribute to the benefits described above. DPA has not been studied in human subjects, however, there are a small number of studies on DPA in cell culture and animals ${ }^{(1)}$. A major reason DPA has not been studied is because it has not been available pure in sufficient quantity to conduct long-term studies on human subjects or animals.

We have data from cell culture and rodent studies which show that DPA is converted to EPA. FAO liver cells were incubated with $50 \mu \mathrm{M}$ DPA for up to $24 \mathrm{~h}$ after which the phospholipid fraction was analysed for fatty acid composition. There was evidence of the appearance of EPA in the cells incubated with DPA by $8 \mathrm{~h}(3.1 \%$ of cell phospholipid fatty acids) and by $24 \mathrm{~h}$ the proportion reached $15.9 \%$. Incubation of the cells with EPA led to the appearance of DPA (10.2\% of fatty acids). There was no appearance of DHA in this time in the case of either the EPA or DPA treated cells.

In young rats ( $n$ 8/group), fed $50 \mathrm{mg}$ of DPA/day orally for $7 \mathrm{~d}$, there was a significant increase in EPA in liver, heart, adipose and skeletal muscle, but not in brain ${ }^{(2)}$. There was also a small but significant increase in the DHA concentration in liver $(P<0.05)^{(2)}$. Rats fed with $50 \mathrm{mg} / \mathrm{d}$ of EPA in the same study, also showed a similar increase in the concentration of DHA in the liver $(P<0.05)$. The conversion of DPA to EPA is described as retroconversion and is thought to involve peroxisomal acyl-CoA oxidase and one cycle of beta-oxidation in peroxisomes $^{(3)}$.

In a second rat study, rats were given an oral dose of either $1-{ }^{14} \mathrm{C}$ labelled EPA, DPA or DHA ( $n$ 5/group) and then placed in a metabolic chamber to collect the expired radiolabelled carbon dioxide over the next $6 \mathrm{~h}$. It was found that significantly more $\mathrm{CO}_{2}$ was collected from rats fed the EPA $(19.3 \pm 1.1 \%$ of the dose) compared with those fed DPA $(5.1 \pm 1.1 \%)$ and DHA $(7.0 \pm 0.4 \%, P<0.05)$. In this study, there was significantly more radioactivity recovered in lipids of liver, brain and kidney for the DHA group compared with the EPA and DPA groups $(P<0.05)$. In heart and skeletal muscle, the incorporation of the radioactivity into lipids was significantly lower in the EPA group compared with DPA and DHA groups $(P<0.05)$.

These novel data indicate that further studies of the role of DPA in mammals should be conducted.

1. Kaur G, Cameron-Smith D, Garg M et al. (2010) Docosapentaenoic acid (22:5n-3): a review of its biological effects. Prog Lipid Res 50, $28-34$.

2. Kaur G, Begg DP, Barr D et al. (2010). Short-term docosapentaenoic acid (22:5n-3) supplementation increases tissue docosapentaenoic acid, DHA and EPA concentrations in rats. Br J Nutr 103, 32-37.

3. Christensen E, Woldseth B, Hagve TA et al. (1993) Peroxisomal beta-oxidation of polyunsaturated long chain fatty acids in human fibroblasts. The polyunsaturated and the saturated long chain fatty acids are retroconverted by the same acyl-CoA oxidase. Scand J Clin Lab Invest Suppl 215, 61-74. 